\title{
The modern trauma pancreaticoduodenectomy for penetrating trauma: a propensity-matched analysis
}

\author{
A. Grigorian ${ }^{1}$ - A. R. Dosch ${ }^{1}$ - P. T. Delaplain ${ }^{1}$ - D. Imagawa ${ }^{1}$ - Z. Jutric ${ }^{1} \cdot$ R. F. Wolf ${ }^{1}$ - D. Margulies ${ }^{2} \cdot$ J. Nahmias $^{1} \mathbb{C}$
}

Received: 20 May 2020 / Accepted: 21 July 2020 / Published online: 26 July 2020

(C) Italian Society of Surgery (SIC) 2020

\begin{abstract}
Trauma pancreaticoduodenectomy (TP) remains a challenging operation with morbidity and mortality rates as high as $80 \%$ and 50\%. Many trauma surgeons consider it surgical dogma to avoid performing a TP during the index operation for patients with severe pancreatic or duodenal injuries. However, there is no modern analysis evaluating this belief. Therefore, we hypothesized no difference in risk of mortality between patients with severe pancreatic or duodenal injury undergoing a TP for penetrating trauma to propensity-matched controls undergoing laparotomy without TP. The Trauma Quality Improvement Program (2010-2016) was queried for adults with severe penetrating pancreatic or duodenal injuries undergoing laparotomy. A 1:2 propensity-matching including demographics/comorbidities, injury severity score, vitals on admission, Glasgow Coma Scale and concomitant injuries for laparotomy with or without TP was performed. Risk of mortality was reported using a univariable logistic regression model. Of 2182 patients with severe pancreatic or duodenal injuries undergoing laparotomy, 54 (2.5\%) underwent TP and 2128 (97.5\%) underwent laparotomy without TP. There were no differences in propensitymatching characteristics. Patients undergoing TP had a similar mortality rate $(20.0 \%$ vs. $28.7 \%, p=0.302)$ but a longer length of stay (LOS) (27.5 vs. 16.5 days, $p=0.017)$. The TP group had a similar associated risk of mortality $(\mathrm{OR}=0.62, p=0.302)$ but higher risk of major complications (OR 3.44, CI 1.35-17.47, $p=0.015$ ). In appropriately selected penetrating trauma patients with severe pancreatic/duodenal injuries, TP is associated with a similar risk of mortality compared to laparotomy without TP. However, TP patients did have an increased associated risk of major complications and longer LOS.
\end{abstract}

Keywords Trauma $\cdot$ Whipple $\cdot$ Pancreaticoduodenectomy $\cdot$ Mortality

\section{Introduction}

Pancreaticoduodenectomy in the setting of trauma remains a challenging and rare operation, occurring in less than $5 \%$ of cases of severe pancreatic head or duodenal injury [1,2]. In contrast to patients with adenocarcinoma in the head of

Presentation: This work won best resident paper from the Southern California caucus at the 91st Annual Meeting of the Pacific Coast Surgical Association, Carlsbad, California, February 2020.

J. Nahmias

jnahmias@uci.edu

1 Division of Trauma, Burns and Surgical Critical Care, Department of Surgery, University of California, Irvine, 333 The City Blvd West, Suite 1600, Orange, CA 92868-3298, USA

2 Division of Trauma, Burns and Surgical Critical Care, Department of Surgery, Cedars-Sinai Hospital, Los Angeles, CA, USA the pancreas, trauma patients are younger and often with less comorbidities. Outside of the challenges of dealing with active hemorrhage, the trauma pancreaticoduodenectomy (TP) operation may be easier to perform since inflammation, desmoplastic reactions and fibrosis secondary to cancer have not disrupted the anatomic planes between the pancreas and major venous structures and furthermore dissection may already be partly accomplished due to the trauma mechanism of injury (i.e. gunshot wound) [3]. Furthermore, the trauma population generally has more physiologic reserve and are void of the chronic debility associated with cancer [4]. However, little is known about the modern TP. In fact, the Eastern Association for the Surgery of Trauma references a lack of data on TP with only a small number of single-center retrospective studies published in the past two decades [5-7].

The only published national analysis on TP analyzed data from a decade ago and reported the only independent predictor of mortality to be the injury severity score (ISS), 
which is calculated post-surgery and thus does not provide much prognostic value to the operating trauma surgeon [8]. Furthermore, they included patients with both blunt and penetrating mechanisms of injury. Pancreatic injury after blunt trauma is rarely a solitary injury with more than $90 \%$ of cases involving injury to at least one other organ including traumatic brain injury (TBI) in up to $50 \%[9,10]$.

Recently trauma patients have benefited from improved pre-hospital transport and damage control surgery $[11,12]$. In addition, balanced resuscitation and permissive hypotension have also led to fewer deaths due to hemorrhage in trauma patients presenting in extremis [13-15]. This may facilitate improved outcomes associated with TP compared to historical reports. However, TP has failed to gain wide popularity among trauma surgeons likely due to reports of morbidity and mortality rates up to $80 \%$ and $50 \%$, respectively [16-19]. However, the national trend for mortality after elective pancreaticoduodenectomy has improved from 25 to below 4\%; a trend partly attributed to the advent of minimally invasive salvage techniques such as interventional radiology, endoscopic retrograde cholangiopancreatography, and improved critical care [20-22]. Therefore, we hypothesized no difference in risk of mortality between contemporary penetrating trauma patients with severe pancreatic or duodenal injury undergoing a TP to propensity-matched controls undergoing laparotomy without TP.

\section{Methods}

A retrospective analysis of the Trauma Quality Improvement Program (TQIP) was performed between January 2010 and December 2016. All patients $\geq 18$-year old presenting with penetrating pancreatic and/or duodenal injuries were identified using the International Classification of Diseases version-9 (ICD-9) diagnosis codes: 863.21, 863.31, 863.81-863.84 and 863.91-863.94. TQIP does not include the American Association for the Surgery of Trauma (AAST) organ injury scale (OIS) for pancreatic injuries. In lieu of this, we used abbreviated-injury-scale (AIS) codes to parallel OIS grades as previously reported by other authors $[23,24]$. We selected only patients with grade-3/4 pancreatic and/or duodenal injuries as these would be the most likely patients to require TP during the index operation. The two compared groups were patients undergoing exploratory laparotomy with and without TP, defined by the ICD-9 procedure codes: 52.7 (TP) and 54.11 (exploratory laparotomy). Only operations occurring within the first $6 \mathrm{~h}$ of admission were included. The primary outcome was in-hospital mortality.

Due to the observed imbalance in the sample size between these two groups, TP patients were matched with patients undergoing exploratory laparotomy without TP using a propensity score model. The propensity score calculated for each observed case is a measure of the likelihood that a patient would have undergone TP. The propensity score used in our analysis was derived from a logistic regression model in which the dependent variable was TP. The variables we used in our model included age, gender, ISS, vitals on admission, use of massive transfusion protocol ( $>6$ units of packed red blood cells within $4 \mathrm{~h}$ or $>10$ units of packed red blood cells within $24 \mathrm{~h}$ ), pre-hospital comorbidities and associated injuries. Patients with similar propensity scores were matched in a 1:2 ratio to compare outcomes among patients that underwent TP and patients that underwent exploratory laparotomy without TP. We included in our analysis only those cases that were within 0.001 of the estimated logit. This technique of defining the closeness of a matched case is termed caliper matching and is the validated method of emulating randomization in observational studies [25]. For this study, we used caliper widths from 0.1 to 0.8 of the pooled standard deviation of the logit of the propensity score, in increments of 0.1. We have used this methodology as Monte Carlo simulations indicate that matching using a caliper width of 0.2 of the pooled standard deviation of the logit of the propensity score affords superior performance in the model. There were no differences in propensity-matching characteristics among the two groups.

Other measured outcomes included total hospital length of stay (LOS), intensive care unit (ICU) LOS, ventilator days, and in-hospital complications including acute respiratory distress syndrome (ARDS), unplanned ICU admission, pneumonia, cerebrovascular accident (CVA), myocardial infarction (MI), acute kidney injury (AKI), deep vein thrombosis (DVT) and pulmonary embolism (PE). Patients undergoing other abdominal operations were also identified including bowel resection, suture laceration of duodenum, subtotal or distal pancreatectomy or other repair of the pancreas. These were defined by the appropriate ICD-9 procedure codes. We defined major complications to include: ARDS, unplanned ICU admission/readmission, pneumonia, CVA or AKI.

All variables were coded as present or absent. Descriptive statistics were performed for all variables. Mann-Whitney $U$ test was used to compare continuous variables and Chi square was used to compare categorical variables for bivariate analysis. Categorical data was reported as percentages, and continuous data was reported as medians with interquartile range or as means with standard deviation. In addition, we also used a Chi square test to compare the mortality rate in our analysis to the one reported in a previous national analysis from 2008 to 2010 [8].

We used a univariable logistic regression model to determine risk of mortality and major in-hospital complications, defined by the development of acute respiratory distress syndrome, unplanned ICU admission, pneumonia, cerebrovascular accident and acute kidney injury. We also 
performed a multivariable logistic regression analysis to report the risk of venous thromboembolism (VTE) disease after adjusting for injuries to the hepatic veins and inferior vena cava. In addition, we performed a multivariable logistic regression analysis on the original dataset (nonpropensity-matched sample) to determine risk of mortality for TP vs. non-TP patients to ensure that the removal of some patients by our propensity-matching methodology did not skew our findings. Predictors of outcome were reported with an odds ratio (OR) with $95 \%$ confidence intervals (CI). Differences with $p<0.05$ were considered statistically significant. All statistical analyses were performed with IBM SPSS Statistics for Windows, Version 24. (Armonk, NY: IBM Corp).

\section{Results}

\section{Demographics of patients with severe pancreatic and duodenal injury undergoing surgery}

Of 2182 patients with penetrating grade-3/4 pancreatic and/ or duodenal injuries undergoing surgery, 54 (2.5\%) underwent TP and 2128 (97.5\%) underwent laparotomy without TP. After propensity-score matching, 40 patients undergoing TP were compared to 80 patients undergoing laparotomy without TP. There were 14 patients that did not fit into our propensity-matched model and thus were excluded from analysis. There were no differences among the two groups with respect to age, gender, ISS, vitals on admission, prehospital comorbidities, massive transfusion and associated injuries.

The TP group had a median age of 28 years and included mostly males (87.5\%). The most common comorbidity was smoking (25.0\%). More patients had an injury to the pancreas $(72.5 \%)$ than to the duodenum (57.5\%) and 57.5\% had a combined injury. The median ISS was 25 , Glasgow Coma Scale score was 15 and 20\% were hypotensive on admission. The most common mechanism was gunshot-wound $(87.5 \%)$ and the most common associated injury was to the liver $(60.0 \%)$. The median time to TP was $43.2 \mathrm{~min}$ and the median time to laparotomy without TP was $42.0 \mathrm{~min}$ (Table 1). More patients in the TP cohort had an associated hepatic vein injury $(5.0 \%$ vs. $0 \%, p=0.044)$ (Table 2$)$. The most commonly performed operation in those not undergoing TP was partial or total colectomy (40.0\%) followed by small intestinal resection (25.0\%) and suture laceration of the duodenum (22.5\%) (Table 3 ). In regard to the pancreas, the most common procedures were pancreas anastomosis (17.5\%), "other" pancreas repair (10.0\%) and distal pancreatectomy (7.6\%). $80 \%$ of the TPs were performed at a Level-I trauma center.

\section{Other clinical outcomes in patients with severe pancreatic and duodenal injury undergoing surgery}

Compared to patients undergoing laparotomy without TP, the TP cohort had a longer hospital LOS (median 27.5 vs. 16.5 days, $p=0.017$ ) but similar ICU LOS (median 13 vs. 6 , $p=0.12$ ) and ventilator days (median 10 vs. $4.0, p=0.06$ ). The TP group also had a higher rate of DVT $(20.0 \%$ vs. $2.5 \%, p=0.001)$ but similar rates of ARDS (2.5\% vs. $1.3 \%$, $p=0.61)$, pneumonia $(15.0 \%$ vs. $5.0 \%, p=0.06)$, AKI $(12.5 \%$ vs. $5.0 \%, p=0.14)$ and unplanned return to the operating room $(2.2 \%$ vs. $10.9 \%, p=0.07)$ compared to the laparotomy without TP cohort. The mortality rate was statistically similar among both groups (TP 20.0\%, no-TP $28.7 \%, p=0.30$ ) (Table 4). Although the mortality rate for TP patients in our contemporary study is lower than a prior 2008-2010 national analysis of 39 patients with TP, however this did not reach statistical significance $(20.0 \%$ vs. $33.0 \%$, $p=0.19)$ [8].

\section{Analysis for risk of mortality and complications}

After adjusting for injuries to the inferior vena cava or hepatic veins, patients undergoing TP had a higher associated risk of VTE compared to patients undergoing laparotomy without $\mathrm{TP}(\mathrm{OR}=4.86, \mathrm{CI}=1.35-17.47, p=0.015)$. On univariable analysis, the risk of major complication was higher in the TP group $(\mathrm{OR}=3.44, \mathrm{CI}=1.44-8.18$, $p=0.005$ ) but the risk of mortality was similar compared to those undergoing laparotomy without $\mathrm{TP}(\mathrm{OR}=0.62$, $p=0.304$ ). In a separate analysis on all patients (original cohort of 2182 patients), TP patients continued to have a similar risk of mortality compared to patients undergoing laparotomy without TP $(\mathrm{OR}=0.58,0.28-1.20, p=0.140)$ (Tables 5, 6).

\section{Discussion}

This retrospective analysis using seven years of data from the TQIP found the incidence of TP in patients with severe (grade-3 or 4) pancreatic and/or duodenal injury after penetrating trauma to be just over $2 \%$. In support of our hypothesis, there was no difference in the risk of mortality between patients who underwent TP during the index operation compared to patients who underwent laparotomy without TP. Furthermore, the actual mortality rate, albeit not statistically significant, demonstrated a trend toward lower mortality for patients undergoing TP compared to matched patients undergoing laparotomy without pancreaticoduodenectomy. Additionally, the mortality rate of TP in our study is statistically similar to a prior national analysis but this may be because our study was underpowered to detect 
Table 1 Demographics of 1:2 propensity-matched patients with severe pancreas/duodenal injury undergoing exploratory laparotomy versus exploratory laparotomy with trauma pancreaticoduodenectomy (TP)

\begin{tabular}{|c|c|c|c|}
\hline Characteristic & $\begin{array}{l}-\mathrm{TP} \\
(n=80)\end{array}$ & $\begin{array}{l}+\mathrm{TP} \\
(n=40)\end{array}$ & $p$ value \\
\hline Age, year, median (IQR) & $28(15)$ & $29(21)$ & 0.846 \\
\hline Male, $n(\%)$ & $70(87.5 \%)$ & $36(90.0 \%)$ & 0.688 \\
\hline ISS, median (IQR) & $19(11)$ & $25(9)$ & 0.732 \\
\hline SBP on admission, median (IQR) & $120(55)$ & $114(46)$ & 0.682 \\
\hline Hypotensive on admission, $n(\%)$ & $16(20.0 \%)$ & $8(20.0 \%)$ & 1.00 \\
\hline Pulse on admission, median (IQR) & $101(37)$ & $99.5(38)$ & 0.942 \\
\hline Tachycardic on admission, $n(\%)$ & $40(50.0 \%)$ & $18(45.0 \%)$ & 0.605 \\
\hline Respiratory rate on admission, median (IQR) & $20(8)$ & $20(7)$ & 0.888 \\
\hline Tachypnea on admission, $n(\%)$ & $27(33.8 \%)$ & $12(30.0 \%)$ & 0.679 \\
\hline GCS on admission, median (IQR) & $15(1)$ & $15(4)$ & 0.981 \\
\hline Massive transfusion, $n(\%)$ & $11(13.8 \%)$ & $5(12.5 \%)$ & 0.849 \\
\hline \multicolumn{4}{|l|}{ Mechanism, $n(\%)$} \\
\hline Stab wound & $5(6.3 \%)$ & $2(5.0 \%)$ & 0.783 \\
\hline Gunshot wound & $70(87.5 \%)$ & $35(87.5 \%)$ & 1.00 \\
\hline Other penetrating & $5(6.3 \%)$ & $3(7.5 \%)$ & 0.304 \\
\hline \multicolumn{4}{|l|}{ Comorbidities, $n(\%)$} \\
\hline Congestive heart failure & 0 & 0 & - \\
\hline Smoker & $20(25.0 \%)$ & $10(25.0 \%)$ & 1.00 \\
\hline End-stage renal disease & 0 & 0 & - \\
\hline Diabetes & $3(3.8 \%)$ & $1(2.5 \%)$ & 0.719 \\
\hline Hypertension & $7(8.8 \%)$ & $3(7.5 \%)$ & 0.815 \\
\hline COPD & $4(5.0 \%)$ & $1(2.5 \%)$ & 0.518 \\
\hline \multicolumn{4}{|l|}{ Pancreaticoduodenal injury, $n(\%)$} \\
\hline Pancreas & $64(80.0 \%)$ & $29(72.5 \%)$ & 0.354 \\
\hline Duodenum & $56(70.0 \%)$ & $23(57.5 \%)$ & 0.174 \\
\hline Combined & $47(58.8 \%)$ & $23(57.5 \%)$ & 0.896 \\
\hline Minutes to procedure, median (IQR) & $42.0(18.9)$ & $43.2(34.8)$ & 0.953 \\
\hline
\end{tabular}

Defined by an abbreviated injury scale grade of 3 or higher

$I S S$ injury severity score, IQR interquartile range, $S B P$ systolic blood pressure, GCS Glasgow Coma Scale, $C O P D$ chronic obstructive pulmonary disease, $A I S$ abbreviated injury scale a difference. In addition, we found the TP group to have a higher rate and associated risk of major in-hospital complications. And finally, the risk of VTE was also significantly higher for those undergoing TP even after adjusting for the increased LOS.

The application of pancreaticoduodenectomy to trauma patients is not novel. Kerry was the first to report the use of pancreaticoduodenectomy in a trauma patient in 1962 [26]. Several years later Foley first reported performing TP as part of the index operation in trauma [27]. The largest case series of TP from a single center includes only 19 patients over a 22 -year period [1]. Our study includes the largest number of patients reported in a single series and attempted to compare similarly matched patients undergoing laparotomy, thus improving generalizability.

Much of what we know about pancreaticoduodenectomy stems from research done on patients undergoing elective surgery for adenocarcinoma in the head of the pancreas. In recent years, patients undergoing pancreaticoduodenectomy in this circumstance are reported to have a less than $4 \%$ perioperative mortality rate-which has decreased considerably from the $25 \%$ perioperative mortality rate reported when Allen Whipple first introduced the operation [22]. Part of the improved mortality is attributed to ancillary staff/services including diagnostic and interventional radiologists, infectious disease specialists, critical care, specialized nurses and dieticians/nutritional support [21]. Additionally, institutional diagnostic/treatment protocols, clinical pathways and standardized post-operative courses are key components in the care of patients undergoing elective pancreaticoduodenectomy at centers specializing in the operation [28]. Much of these features are inherent to trauma centers and systems. Furthermore, the care of the trauma patient is rooted by a multidisciplinary approach including interventional radiologists (IR) and gastroenterologists as integral members of the trauma team [29, 30]. In fact, the American 
Table 2 Injuries of 1:2 propensity-matched patients with severe pancreas/duodenal injury undergoing exploratory laparotomy versus exploratory laparotomy with trauma pancreaticoduodenectomy (TP)

\begin{tabular}{llll}
\hline Characteristic & $\begin{array}{l}-\mathrm{TP} \\
(n=80)\end{array}$ & $\begin{array}{l}+\mathrm{TP} \\
(n=40)\end{array}$ & $p$ value \\
\hline Vascular Injuries, $n(\%)$ & & & \\
Aorta & $8(10.0 \%)$ & $1(2.5 \%)$ & 0.141 \\
Celiac artery & 0 & $1(2.5 \%)$ & 0.156 \\
Superior mesenteric artery & $6(7.5 \%)$ & $4(10.0 \%)$ & 0.640 \\
Inferior mesenteric artery & 0 & 0 & - \\
Inferior vena cava & $21(26.3 \%)$ & $14(35.0 \%)$ & 0.320 \\
Hepatic vein & 0 & $2(5.0 \%)$ & $\mathbf{0 . 0 4 4}$ \\
Portal vein & $1(1.3 \%)$ & $2(5.0 \%)$ & 0.215 \\
Associated Injuries, $n(\%)$ & & & \\
Traumatic brain injury & $2(2.5 \%)$ & $1(2.5 \%)$ & 1.00 \\
Spine & $14(17.5 \%)$ & $8(20.0 \%)$ & 0.739 \\
Pelvis fracture & $3(3.8 \%)$ & $1(2.5 \%)$ & 0.719 \\
Upper extremity fracture & $8(1.0 \%)$ & $3(7.5 \%)$ & 0.655 \\
Lower extremity fracture & $9(11.3 \%)$ & $3(7.5 \%)$ & 0.519 \\
Lung & $10(12.5 \%)$ & $7(17.5 \%)$ & 0.459 \\
Heart & 0 & 0 & - \\
Stomach & $34(42.5 \%)$ & $15(37.5 \%)$ & 0.599 \\
Colorectal & $32(40.0 \%)$ & $20(50.0 \%)$ & 0.297 \\
Liver & $42(52.5 \%)$ & $24(60.0 \%)$ & 0.436 \\
Spleen & $6(7.5 \%)$ & $2(5.0 \%)$ & 0.605 \\
Kidney & $26(32.5 \%)$ & $12(30.0 \%)$ & 0.781 \\
\hline Define & &
\end{tabular}

Defined by an abbreviated injury scale grade of 3 or higher

Table 3 Other procedures in 1:2 propensity-matched patients with severe pancreas/duodenal injury undergoing exploratory laparotomy without trauma pancreaticoduodenectomy (TP)

\begin{tabular}{lr}
\hline Procedure & $\%$ \\
\hline Partial or total colectomy & 40.0 \\
Other small intestine resection & 25.0 \\
Suture laceration of duodenum & 22.5 \\
Anastomosis pancreas & 17.5 \\
Other gastroenterostomy & 13.0 \\
Other pancreas repair & 10.0 \\
Distal pancreatectomy & 7.6 \\
Proximal pancreatectomy & 7.5 \\
Subtotal pancreatectomy & 5.0 \\
\hline
\end{tabular}

College of Surgeon's mandates that verified trauma centers have an institutionally agreed upon response time from interventional radiology teams [31]. The evolving role of IR, interventional gastroenterology and the "built-in" treatment pathways of trauma care may help explain why we found the modern TP to have a similar risk of mortality compared to a trauma laparotomy for similarly injured patients and why the perioperative mortality rate is far less than the $50 \%$ reported
Table 4 Clinical outcomes of 1:2 propensity-matched patients with severe pancreas/duodenal injury undergoing exploratory laparotomy versus exploratory laparotomy with trauma pancreaticoduodenectomy (TP)

\begin{tabular}{llll}
\hline Outcome & $\begin{array}{l}-\mathrm{TP} \\
(n=80)\end{array}$ & $\begin{array}{l}+\mathrm{TP} \\
(n=40)\end{array}$ & $p$ value \\
\hline LOS, days, median (IQR) & $16.5(25)$ & $27.5(35)$ & $\mathbf{0 . 0 1 7}$ \\
ICU, days, median (IQR) & $6.0(8)$ & $13.0(24)$ & 0.125 \\
Ventilator, days, median (IQR) & $4.0(7)$ & $10.0(13)$ & 0.063 \\
Major complications, $n(\%)$ & & & \\
ARDS & $1(1.3 \%)$ & $1(2.5 \%)$ & 0.614 \\
Unplanned ICU admission & $6(7.5 \%)$ & $4(10.0 \%)$ & 0.640 \\
Pneumonia & $4(5.0 \%)$ & $6(15.0 \%)$ & 0.062 \\
Cerebrovascular accident & 0 & $1(2.5 \%)$ & 0.156 \\
Myocardial infarction & 0 & 0 & - \\
$\quad$ Acute kidney injury & $4(5.0 \%)$ & $5(12.5 \%)$ & 0.141 \\
Venous thromboembolism, $n(\%)$ & & & \\
Deep vein thrombosis & $2(2.5 \%)$ & $8(20.0 \%)$ & $\mathbf{0 . 0 0 1}$ \\
Pulmonary embolism & $2(2.5 \%)$ & $2(5.0 \%)$ & 0.472 \\
Mortality, $n$ (\%) & $23(28.7 \%)$ & $8(20.0 \%)$ & 0.302 \\
\hline
\end{tabular}

$L O S$ length of stay, $I Q R$ interquartile range, $I C U$ intensive care unit, $A R D S$ acute respiratory distress syndrome, VAP ventilator-associated pneumonia

Table 5 Logistic regression analysis for risk of venous thromboembolism in 1:2 propensity-matched patients with severe pancreas/duodenal injury undergoing exploratory laparotomy and trauma pancreaticoduodenectomy versus exploratory laparotomy alone

\begin{tabular}{llll}
\hline Risk factor & OR & CI & $p$ value \\
\hline Mortality & 0.62 & $0.25-1.55$ & 0.304 \\
Major complications $^{\mathrm{a}}$ & 3.44 & $1.44-8.18$ & 0.005 \\
Venous thromboembolism $^{\mathrm{b}}$ & 4.86 & $1.35-17.47$ & 0.015 \\
\hline
\end{tabular}

${ }^{a}$ Defined by acute respiratory distress syndrome, unplanned intensive care unit admission, pneumonia, cerebrovascular accident, acute kidney injury

${ }^{\mathrm{b}}$ Adjusted for inferior vena cava and hepatic vein injury

in the first several case-series' of trauma patients undergoing TP $[16,19]$. This also helps explain the fact that while more of our trauma patients survived, we did find that TP patients had a higher risk of major complications compared to similar patients undergoing laparotomy. This continued high rate/risk of complications is mirrored in the elective pancreaticoduodenectomy literature with complication rates as high as $45 \%$ [20,32]. Additionally, the low rate of unplanned returns to the operating room found in this study support the fact that complications can often be dealt with in a more minimally invasive manner thus improving mortality outcomes. Future prospective studies should capture the rate of endoscopic (i.e. sphincterotomy and/or pancreatic/biliary stent placement) and/or IR-based interventions 
Table 6 Adjusted multivariable logistic regression analysis for risk of mortality in all severe pancreas/duodenal injured patients undergoing exploratory laparotomy and pancreaticoduodenectomy versus exploratory laparotomy alone

\begin{tabular}{lllr}
\hline Risk factor & OR & CI & $p$ value \\
\hline Pancreaticoduodenectomy & 0.58 & $0.28-1.20$ & 0.140 \\
Combined pancreas/duodenum injury & 1.75 & $1.32-2.31$ & $<0.001$ \\
Hypotension on admission & 2.39 & $1.84-3.09$ & $<0.001$ \\
Tachycardia on admission $^{\text {Severe }}{ }^{\text {a AIS-head }}$ & 1.53 & $1.23-1.92$ & $<0.001$ \\
Severe $^{\text {a }}$ AIS-thorax & 2.82 & $1.40-5.67$ & 0.004 \\
Severe $^{\text {a }}$ AIS-abdomen & 1.98 & $1.39-2.83$ & $<0.001$ \\
Injury severity score $\geq 25$ & 2.65 & $1.97-3.57$ & $<0.001$ \\
\hline
\end{tabular}

Defined by an abbreviated injury scale grade of 3 or higher AIS abbreviated injury scale

${ }^{a}$ Adjusted for combined grade 3/4 pancreatic and duodenal injuries

(angioembolization and/or image-guided drainage of peripancreatic fluid collections) to definitively determine the utilization rates and effects on outcomes.

Patients undergoing pancreatoduodenectomy have a higher incidence of post-operative VTE. In modern reports, the rate of VTE ranges between 1 and 21\% [33]. Since most of the reports on VTE in pancreatoduodenectomy are based on patients with pancreatic cancer, whom already have a higher risk of VTE, the contribution that the operation itself may have is difficult to ascertain. Additionally, major surgery in and of itself is a risk factor for VTE [34]. However, in our study we compared similarly injured patients undergoing laparotomy without TP, which is still a major abdominal surgery and found the TP group to have a nearly eight-fold higher associated risk of VTE. Stasis or injury of the portal venous system which may occur during dissection of the pancreatic head and porta hepatis may partly explain the increased risk of VTE within trauma patients undergoing TP [33]. Additionally, the increased time it would take to perform a TP compared to a laparotomy without TP may partly explain this finding [20,35]. Further explanation may be related to our finding of a higher rate of IVC injury in the TP group. However, our post-hoc multivariable analysis demonstrated that even after controlling for IVC injury, the risk of VTE continued to be significantly higher in the TP group.

We have several limitations to note in our study. This was an analysis of a large national database with multiple participating trauma centers and as such, we can only report associations. TQIP does not grade injuries using the American Association for the Surgery of Trauma organ injury score (OIS) for pancreatic injuries. We decided to use the available AIS to loosely parallel the OIS realizing this is an imperfect methodology. Additionally, reporting bias and coding errors are undoubtedly present. We also were missing important information such as details of and location of pancreatic (relationship to mesenteric vessels and ductal involvement) and duodenal (relationship to ampulla and wall circumference of injury) injuries, as well as pancreatic/biliary post-surgical complications (i.e. pancreatic leak and/or bile leak) and what post-operative interventional services were utilized. Since the concomitant injuries were coded using ICD-9 codes, it is unknown if the IVC injuries were simply radiographic evidence of injury to the vessel, an injury identified intraoperatively or an injury with associated massive hemorrhage. Other missing information include subsequent liver failure, type of pancreatic reconstruction and information regarding intraoperative drain placement. We also do not have any long-term data regarding TP reconstruction complications as well as quality of life evaluations as this database only includes outcomes pertaining to the index hospitalization. Furthermore, this study only included those receiving a surgery within the first $6 \mathrm{~h}$ of admission. As such, we are not able to report on patients undergoing delayed reconstruction after an initial damage control surgery. Finally, and most notably, there is also almost certainly a selection bias for patients selected to undergo TP, as such the authors are not suggesting increased use of TP. We are more reporting that in appropriately selected patients, TP may be a reasonable option and no longer carries the extreme mortality rate historically reported. However, future multicenter prospective studies are needed to evaluate these findings.

\section{Conclusion}

The use of TP in penetrating trauma patients continues to be rare. In appropriately selected trauma patients with severe pancreatic/duodenal injuries, TP is associated with a similar risk of mortality as laparotomy without TP. However, TP patients did have a longer LOS as well as an increased associated risk of major complications and VTE.

Funding No funding was received for this work.

\section{Compliance with ethical standards}

Conflict of interest The authors declare that they have no conflicts of interest.

Research involving human participants and/or animals This research involved humans. However, since this retrospective study was performed using a national database with deidentified patients, risk toparticipants is minimal.

Informed consent There is no consent required. 


\section{References}

1. Krige JE, Nicol AJ, Navsaria PH (2014) Emergency pancreatoduodenectomy for complex injuries of the pancreas and duodenum. HPB (Oxford) 16(11):1043-1049. https://doi.org/10.1111/ hpb. 12244

2. Antonacci N, Di Saverio S, Ciaroni V, Biscardi A, Giugni A, Cancellieri F, Coniglio C, Cavallo P, Giorgini E, Baldoni F, Gordini G, Tugnoli G (2011) Prognosis and treatment of pancreaticoduodenal traumatic injuries: which factors are predictors of outcome? J Hepatobiliary Pancreat Sci 18(2):195-201. https:// doi.org/10.1007/s00534-010-0329-6

3. Apte MV, Park S, Phillips PA, Santucci N, Goldstein D, Kumar RK, Ramm GA, Buchler M, Friess H, McCarroll JA, Keogh G, Merrett N, Pirola R, Wilson JS (2004) Desmoplastic reaction in pancreatic cancer: role of pancreatic stellate cells. Pancreas 29(3):179-187

4. Melis M, Marcon F, Masi A, Pinna A, Sarpel U, Miller G, Moore H, Cohen S, Berman R, Pachter HL (2012) The safety of a pancreaticoduodenectomy in patients older than 80 years: risk vs. benefits. HPB 14(9):583-588

5. Thompson CM, Shalhub S, DeBoard ZM, Maier RV (2013) Revisiting the pancreaticoduodenectomy for trauma: a single institution's experience. J Trauma Acute Care Surg 75(2):225-228. https ://doi.org/10.1097/TA.0b013e31829a0aaf

6. Asensio JA, Petrone P, Roldan G, Kuncir E, Demetriades D (2003) Pancreaticoduodenectomy: a rare procedure for the management of complex pancreaticoduodenal injuries. J Am Coll Surg 197(6):937-942. https://doi.org/10.1016/j.jamcollsur g.2003.07.019

7. Bokhari F, Phelan H, Holevar M, Brautigam R, Collier B, Como J (2009) EAST guidelines for the diagnosis and management of pancreatic trauma. Eastern Association for the Surgery of Trauma, Chicago

8. van der Wilden GM, Yeh DD, Hwabejire JO, Klein EN, Fagenholz PJ, King DR, de Moya MA, Chang Y, Velmahos GC (2014) Trauma Whipple: do or don't after severe pancreaticoduodenal injuries? An analysis of the National Trauma Data Bank (NTDB). World J Surg 38(2):335-340

9. Cirillo RL, Koniaris LG (2002) Detecting blunt pancreatic injuries. J Gastrointest Surg 6(4):587-598

10. Becker A, Peleg K, Olsha O, Givon A, Kessel B, Bahouth H, Becker A, Hadary A, Jeroukhimov M, Karawani I (2018) Analysis of incidence of traumatic brain injury in blunt trauma patients with Glasgow Coma Scale of 12 or less. Chin J Traumatol 21(3):152-155

11. Swaroop M, Straus DC, Agubuzu O, Esposito TJ, Schermer CR, Crandall ML (2013) Pre-hospital transport times and survival for Hypotensive patients with penetrating thoracic trauma. J Emerg Trauma Shock 6(1):16-20. https://doi.org/10.4103/09742700.106320

12. Rotondo MF, Schwab CW, McGonigal MD, Phillips GR 3rd, Fruchterman TM, Kauder DR, Latenser BA, Angood PA (1993) 'Damage control': an approach for improved survival in exsanguinating penetrating abdominal injury. J Trauma 35(3):375-382 (discussion 382-373)

13. Holcomb JB, Del Junco DJ, Fox EE, Wade CE, Cohen MJ, Schreiber MA, Alarcon LH, Bai Y, Brasel KJ, Bulger EM (2013) The prospective, observational, multicenter, major trauma transfusion (PROMMTT) study: comparative effectiveness of a time-varying treatment with competing risks. JAMA Surg 148(2):127-136

14. Holcomb JB, Tilley BC, Baraniuk S, Fox EE, Wade CE, Podbielski JM, del Junco DJ, Brasel KJ, Bulger EM, Callcut RA, Cohen MJ, Cotton BA, Fabian TC, Inaba K, Kerby JD, Muskat P, O'Keeffe T, Rizoli S, Robinson BR, Scalea TM, Schreiber
MA, Stein DM, Weinberg JA, Callum JL, Hess JR, Matijevic N, Miller CN, Pittet JF, Hoyt DB, Pearson GD, Leroux B, van Belle G (2015) Transfusion of plasma, platelets, and red blood cells in a 1:1:1 vs a 1:1:2 ratio and mortality in patients with severe trauma: the PROPPR randomized clinical trial. JAMA 313(5):471-482. https://doi.org/10.1001/jama.2015.12

15. Cantle PM, Cotton BA (2017) Balanced resuscitation in trauma management. Surg Clin 97(5):999-1014

16. Asensio JA, Demetriades D, Hanpeter DE, Gambaro E, Chahwan S (1999) Management of pancreatic injuries. Curr Probl Surg 36(5):325-419

17. Gentilello LM, Cortes V, Buechter KJ, Gomez GA, Castro M, Zeppa R (1991) Whipple procedure for trauma: is duct ligation a safe alternative to pancreaticojejunostomy? J Trauma 31(5):661667 (discussion 667-668)

18. Lissidini G, Prete FP, Piccinni G, Gurrado A, Giungato S, Prete F, Testini M (2015) Emergency pancreaticoduodenectomy: when is it needed? A dual non-trauma centre experience and literature review. Int J Surg 21:S83-S88

19. Thal A, Wilson R (1964) A pattern of severe blunt trauma to the region of the pancreas. Surg Gynecol Obstet 119:773-778

20. Cusworth BM, Krasnick BA, Nywening TM, Woolsey CA, Fields RC, Doyle MM, Liu J, Hawkins WG (2017) Whipple-specific complications result in prolonged length of stay not accounted for in ACS-NSQIP Surgical Risk Calculator. HPB 19(2):147-153

21. Lieberman MD, Kilburn H, Lindsey M, Brennan MF (1995) Relation of perioperative deaths to hospital volume among patients undergoing pancreatic resection for malignancy. Ann Surg 222(5):638

22. Cameron JL, Riall TS, Coleman J, Belcher KA (2006) One thousand consecutive pancreaticoduodenectomies. Ann Surg 244(1): 10

23. Siboni S, Kwon E, Benjamin E, Inaba K, Demetriades D (2016) Isolated blunt pancreatic trauma: a benign injury? J Trauma Acute Care Surg 81(5):855-859

24. Phillips B, Turco L, McDonald D, Mause E, Walters RW (2018) A subgroup analysis of penetrating injuries to the pancreas in 777 patients from the National Trauma Data Bank, 2010-2014. J Surg Res 225:131-141

25. Austin PC (2011) Optimal caliper widths for propensity-score matching when estimating differences in means and differences in proportions in observational studies. Pharm Stat 10(2):150-161. https://doi.org/10.1002/pst.433

26. Kerry RL, Glas WW (1962) Traumatic injuries of the pancreas and duodenum: a clinical and experimental study. Arch Surg 85(5):813-816

27. Foley WJ, Gaines RD, Fry WJ (1969) Pancreaticoduodenectomy for severe trauma to the head of the pancreas and the associated structures: report of three cases. Ann Surg 170(5):759

28. Gordon TA, Bowman HM, Tielsch JM, Bass EB, Burleyson GP, Cameron JL (1998) Statewide regionalization of pancreaticoduodenectomy and its effect on in-hospital mortality. Ann Surg 228(1):71-78. https://doi.org/10.1097/00000658-19980 7000-00011

29. Pryor JP, Braslow B, Reilly PM, Gullamondegi O, Hedrick JH, Schwab CW (2005) The evolving role of interventional radiology in trauma care. J Trauma 59(1):102-104. https://doi. org/10.1097/01.ta.0000171455.66437.de

30. Yim NY, Kim YT, Kim HO, Kim JK, Kang YJ, Park YC, Park CY (2014) Role of interventional radiology in trauma care: retrospective study from single trauma center experience. Am J Emerg Med 32(11):1315-1318. https://doi.org/10.1016/j.ajem.2014.07.037

31. Trauma ACoSCo (2014) Resources for optimal care of the injured patient. American College of Surgeons, Chicago

32. Pugalenthi A, Protic M, Gonen M, Kingham TP, Angelica MID, Dematteo RP, Fong Y, Jarnagin WR, Allen PJ (2016) 
Postoperative complications and overall survival after pancreaticoduodenectomy for pancreatic ductal adenocarcinoma. J Surg Oncol 113(2):188-193

33. Ansari D, Ansari D, Andersson R, Andrén-Sandberg Å (2015) Pancreatic cancer and thromboembolic disease, 150 years after Trousseau. Hepatobiliary Surg Nutr 4(5):325

34. Kreutzer L, Minami C, Yang A (2016) Preventing venous thromboembolism after surgery. JAMA 315(19):2136-2136

35. Kim JY, Khavanin N, Rambachan A, McCarthy RJ, Mlodinow AS, De Oliveria GS, Stock MC, Gust MJ, Mahvi DM (2015) Surgical duration and risk of venous thromboembolism. JAMA Surg 150(2):110-117. https://doi.org/10.1001/jamasurg.2014.1841

Publisher's Note Springer Nature remains neutral with regard to jurisdictional claims in published maps and institutional affiliations. 